\title{
Identification of putative laryngeal and pharyngeal lichen planus lesions: an endoscopic preliminary evaluation in 16 patients
}

Bulfamante AM*, D’Agostino Fiorenza U*, Castellarin P*, Pipolo C*, Cacioppo G*, Felisati G*, Saibene AM*

Otolaryngology Unit, Santi Paolo e Carlo Hospital, Department of Health Sciences, UniversitàdegliStudi di Milano, Milan, Italy

Corresponding Author

D’Agostino Fiorenza U

umbertodagostinofiorenza@gmail.com

This article has been accepted for publication and undergone full peer review but has not been through the copyediting, typesetting, pagination and proofreading process, which may lead to differences between this version and the Version of Record. Please cite this article as doi: 10.1111/COA.13633

This article is protected by copyright. All rights reserved 
DR UMBERTO D'AGOSTINO FIORENZA (Orcid ID : 0000-0001-9438-3353)

Article type : Correspondence: Our Experience

\section{Keypoints}

1. Lichen planus is a very common autoimmune inflammatory disease, affecting $1-3 \%$ of general population

2. Its oral form - also known as Oral Lichen Planus - is the most common, with a prevalence between $1 \%$ and $2 \%$ in the general population.

3. LP lesions were discovered in the esophagus in OLP patients. The malignant transformation risk of those lesions is currently unknown, but it can be equivalent to oral lesions - approximately 1-3\%.

4. Our study points towards the presence of pharyngolaryngeal lichenoid lesions with unknown neoplastic potential

5. The clinical value of a complete, periodical, upper aerodigestive tract evaluation in lichen planus patients needs to be specifically evaluated

\section{Introduction}

Lichen Planus (LP) is a chronic autoimmune inflammatory disease affecting the stratified squamous epithelium and the lamina propria of skin and mucous membranes. Its oral form - also known as Oral Lichen Planus (OLP) - is the most common, with a prevalence between 1\% and $2 \%$ in the general population, while its incidence is estimated at around $2.2 \%$. OLP affects more often female subjects with a female/male ratio of $2: 1$; it is more frequent in Caucasian individuals, who have a relative risk of around 5.5 compared to other ethnic groups; it has an onset age between 30 and 60, although in rare cases it can occur earlier. The various LP forms are often associated: in patients with OLP diagnosis, $20 \%$ of women and $15 \%$ of men have concomitant genital and/or cutaneous lesions, while in patients with cutaneous LP diagnosis, 
oral lesions occur in $70-77 \%$ of cases $^{2}$. More recently, LP lesions were discovered in the esophagus in OLP patients with dysphagia as concomitant findimg ${ }^{1}$. Early diagnosis of such lesions is extremely important due their potential malignant transformation; the transformation risk is currently unknown, but it can be equivalent to oral lesions - approximately $1-3 \%{ }^{3}$.

Despite the LP lesions ubiquity in almost every mucocutaneous region of the human body, cases of laryngeal or pharyngeal lichen planus were never reported in literature. In this study we examined the upper airways of a pilot group of patients already diagnosed with OLP, discovering lichenoid lesions in the hypopharynx and larynx. Those lesions, in most cases asymptomatic, could represent a potential starting point for a subsequent malignant transformation.

\section{Material and Methods}

\section{ETHICAL CONSIDERATIONS}

Data collection for this study was performed in patients who were already prescribed a combined otolaryngology and oral pathology evaluation, therefore it didn't require any additional diagnostic or therapeutic procedure. The study was therefore approved by the Internal Review Board of ASST Santi Paolo e Carlo, Milan, Italy and there were no ethical concerns of sort.

\section{PATIENTS}

Eligible subjects were all adults over the age of 18, with a previous clinical and histological OLP diagnosis, who were prescribed a combined otolaryngology and oral pathology evaluation in our secondary care outpatient clinic.

The sample group was composed by 16 consecutive patients aged 47-85. Out of them 2 were males $(12.5 \%)$ and 14 females (87.5\%). 4 out 16 patients had a prior diagnosis of laryngopharyngeal reflux (LPR), while 5 of them had a prior histological diagnosis of oral verrucous proliferativa leukoplakia (VPL). Last, 3 out 16 patients were active smokers, none was a former smoker.

\section{CLINICAL EVALUATION}

An oral pathologist and an otolaryngologist examined all subjects at the same time. Each patient was investigated for oral symptoms (pain, swelling or burning sensations) and pharyngeal/laryngeal symptoms such as dysphagia, foreign body sensation, pharyngodynia, dyspnea and dysphonia. 
All patients underwent a conventional oral examination, a narrow band imaging (NBI)-powered oral endoscopy and a fiberoptic examination of the upper aerodigestive tract, with white light and NBI technique, aimed at identify any kind of oral and pharyngolaryngeal LP lesion.

\subsection{Results}

At the time of the visit, 8 subjects reported oral symptoms, while only one patient, already diagnosed with LPR, reported pharyngodinia and foreign body sensation. No patient reported laryngeal symptoms.

All patients in the study had oral lesions at time of examination, with lichenoid reticular striae being the most common type of lesion identified (12 out 16 patients).

During the endoscopic upper aerodigestive tract examinations, lesions were found in 7 patients. No patient had lesions in the sinonasal cavities or rhinopharynx. 6 patients had one or more plaque lesions located on the hypopharyngeal posterior wall (Figure 1 and Figure 2).

Other sites were the right piriform sinus in one patient and the left aryepiglottic plica in another one. For what concerns the larynx, we found a plaque lesion in the interarithenoid space in one patient, while no lesions were identified in the glottic and subglottic larynx (Figure 3 and Figure 4).

No patients reported side effects or traumata during the examinations.

\subsection{Discussion}

\section{KEY FINDINGS}

Our study is the first one in literature to perform an integrated endoscopic evaluation in the follow-up of patients diagnosed with OLP.

Being a pilot study, it is not possible to extrapolate statistically relevant statements or correlations; however, very interesting observations have emerged from a clinical point of view, which should be furthered by larger studies.

The first observation is the presence of pharyngeal lichenoid lesions in asymptomatic patients. In our series the only symptomatic patient had lichenoid plaques on the posterior wall of the hypopharynx, which however overlapped a history of LPR, which could explain symptoms.

From these observations we can deduce that lesions remain hidden in patients with LPO not only because of their localization - often not even taken into account - but also for the lack of 
related symptoms. Given that the same observation emerged from esophageal LP studies ${ }^{1}$, there is a strong need to explore the role of routine upper aerodigestive tract endoscopic examination in LPO in order to identify lesions and monitor them.

Our study shows that the group with pharyngeal lesions has a higher average age than the group without lesions [ $68.57+/-2.26$ vs $66.22+/-4.57]$ and that patients with concomitant diagnosis of VPL don't show higher incidence of pharyngolaryngeal lesions (20\% of VLP patients vs. $54.55 \%$ of patients without VLP) presented no VLP diagnosis. These observations unfortunately - have low statistical relevance due to the small number of patients involved in the study.

\section{PATHOPHYSIOLOGICAL HYPOTHESIS}

The most common site of the lesions found during endoscopy was the hypopharynx. All lesions were found in sites that come in direct contact with the food bolus, and this might represent the decisive factor for developing lichenoid lesions. This hypothesis makes more sense if we consider reactive isomorphism - a typical characteristic of certain dermatoses such as the $\mathrm{LP}^{4}$. The reactive isomorphism - or Koebner phenomenon - consists in the development of clinically and histologically equal lesions to those of the underlying pathology after a trauma of the mucosa or skin $^{5}$. Among the various sites explored through endoscopic examination, the hypopharynx is the most subject to trauma due to the transit of the food bolus, and, in turn, this would also explain why no lesions have been found in nasopharynx and glottic/subglottic larynx.

\section{COMPARISON WITH CURRENT KNOWLEDGE}

The ease with which we were able to find these lichenoid lesions in the upper aerodigestive tract compared with the almost absence of literature on this subject - makes us wonder why these sites have been neglected in LPO patients evaluation. A most probable hypothesis is that otolaryngologists usually evaluate the upper aerodigestive tract, while LPO patients are mostly followed by oral pathologist. Thus a grey area is formed, where the former specialists lack the study population, while the latter usually lack the knowhow on pharyngeal and laryngeal endoscopy ${ }^{6}$.

Another explanation could lie in the lesion size and localization. In our study lesions often appear to be very small and positioned tangentially to the optical fiber lens, making them all the more difficult to be identified without exploiting the NBI technique which in our study allowed to identify lesions that escaped investigation with regular white light.

These observations become even more poignant if we consider how esophageal LP is becoming an increasingly important and well-known clinical entity among gastroenterologists. During an 
esophagoscopy it is theoretically possible to perform an evaluation of the oropharynx and hypopharynx. However while carrying out those exams the specialist often is focused on finding more distal lesions, and therefore in this case a pharyngeal involvement can go unnnoticed ${ }^{7}$.

\section{CLINICAL AND SCIENTIFIC APPLICABILITY OF THE STUDY}

The neoplastic risk of OLP lesions is a consolidated reality: a 2017 data meta-analysis on 20095 patients calculated a risk of $1.1 \%{ }^{8}$. Regarding esophageal lesions, in the retrospective study of Codipilly DC et al. on 132 patients diagnosed with esophageal LP it was shown that within 3 years $6.1 \%$ had developed a squamous-cell carcinoma. This data could be far from the true value of the neoplastic degeneration risk in the pharynx and larynx, but they seem to suggest - along with several case reports ${ }^{21-22-23}$ - that degenerative risk is associated with LP. Unlike the oral cavity and esophagus, there are no studies that correlated pharyngeal LP with squamous-cell carcinoma, probably due to the lack of studies in general: in light of the results of other studies on other anatomical sites ${ }^{8-9-10}$ it would still be wrong to say the opposite.

It comes almost naturally taking into account these considerations, that there is missing point in the management of LPO patients and that the real prevalence of these lesions, obviously still requiring an histologic evaluation, must be better understood and explored in a significant number of patients, along with their potentially risk of neoplastic degeneration.

All this makes us understand that a more interdisciplinary approach and the involvement of otolaryngologists, could guarantee a better evaluation of an LP patient.

\section{Bibliography}

1. Quispel R, van Boxel OS, Schipper ME, Sigurdsson V, Canninga-van Dijk MR, Kerckhoffs A, Smout AJ, Samsom M, Schwartz MP. High prevalence of esophageal involvement in lichen planus: a study using magnification chromoendoscopy. Endoscopy 2009;41:187-193.

2. Parashar P. Oral lichen planus. Otolaryngol Clin North Am 2011;44:89-107.

3. Fox LP, Lightdale CJ, Grossman ME. Lichen planus of the esophagus: What dermatologists need to know. J Am Acad Dermatol 2011;65:175-183. 
4. Kumara L, Rangaraj M, Karthikeyan K. Drawstring lichen planus: A unique case of Koebnerization. Indian Dermatol Online J 2016;7:201-202.

5. Camargo CM, Brotas AM, Ramos-e-Silva M, Carneiro S. Isomorphic phenomenon of Koebner: $\quad$ facts and controversies. Clin Dermatol 2013;31:741-749.

6. Rimkevičius A, Aleksejūnienè J, Pūrienė A, Šeinin D, Rastenienė R. Oral lichen planus: a 4year clinical follow-up study. Turk J Med Sci 2017;47:514-522.

7. Emura F, Baron TH, Gralnek IM. The pharynx: examination of an area too often ignored during upper endoscopy. Gastrointest Endosc 2013;78:143-149.

8. Aghbari SMH, Abushouk AI, Attia A, Elmaraezy A, Menshawy A, Ahmed MS, Elsaadany BA, Ahmed EM. Malignant transformation of oral lichen planus and oral lichenoid lesions: A metaanalysis of 20095 patient data. Oral Oncol 2017;68:92-102.

9. Chryssostalis A, Gaudric M, Terris B, Coriat R, Prat F, Chaussade S. Esophageal lichen planus: a series of eight cases including a patient with esophageal verrucous carcinoma. A case series. Endoscopy 2008;40:764-768.

10. Schwartz MP, Sigurdsson V, Vreuls W, Lubbert PH, Smout AJ. Two siblings with lichen planus and squamous cell carcinoma of the oesophagus. Eur J Gastroenterol Hepatol 2006;18:1111-1115.

Figure Legends 
Figure 1. Endoscopic white light image showing a plaque lesion on the posterior wall of the hypopharynx. Due to its tangent position with respect to the observation point, the lesion is only barely noticeable.

Figure 2. Endoscopic image from the patient in Figure 1. The use of the Narrow Band Imaging technique allows a better contrast, making the plaque lesion on the posterior wall of the hypopharynx more definite and easier to detect.

Figure 3. Endoscopic white light image showing a plaque lesion on the mucosa of the interarytenoid space.

Figure 4. Endoscopic image from the patient in Figure 3. In this patient the use of the Narrow Band Imaging technique allows to rule out any further lesion as well as mucosal superficial vasculature alteration 